\title{
AVALIAÇÃO DO EFEITO dA CARGa DE SÓlIDOS SOBRE A HIDRÓLISE ENZIMÁTICA DO BAGAÇO DE CANA-DE-AÇÚCAR E COMPARAÇÃO ENTRE COMPLEXOS ENZIMÁTICOS COMERCIAIS
}

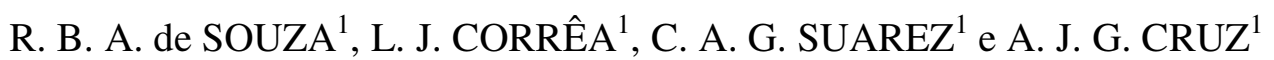 \\ ${ }^{1}$ Universidade Federal de São Carlos, PPGEQ, Programa de Pós-Graduação em Engenharia Química \\ E-mail para contato: renata.beraldo@yahoo.com.br
}

\begin{abstract}
RESUMO - A hidrólise enzimática é uma das principais etapas na produção do etanol de segunda geração. O objetivo desse trabalho foi avaliar o efeito da carga de sólidos na hidrólise do bagaço da cana-de-açúcar e comparar o desempenho de dois complexos enzimáticos comerciais. A biomassa foi pré-tratada hidrotermicamente $\left(195^{\circ} \mathrm{C}, 10 \mathrm{~min}\right.$.,

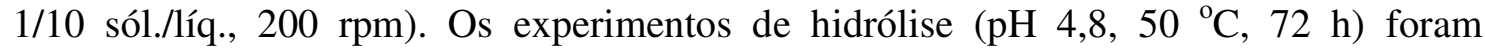
realizados em frascos de Erlenmeyer (volume de meio reacional $50 \mathrm{~mL}$ ). As cargas de sólido avaliadas foram 1015 e $20 \%$. Dois complexos enzimáticos comerciais, denominados complexo-A e complexo-B, foram avaliados. A mesma carga enzimática foi empregada em todos os ensaios (20 FPU/ $\left.\mathrm{g}_{\text {bagaço }}\right)$. Em $72 \mathrm{~h}$ de hidrólise as conversões de celulose foram: complexo-A: 33,5\% (10\% sólidos), 24,8\% (15\% de sólidos) e 18,1\% (20\% de sólidos); complexo-B: 60,7\% (10\% de sólidos), 61,8\% (15\% de sólidos) e 42,4\% (20\% de sólidos). O complexo-B foi mais eficiente obtendo-se maiores conversões e possibilitando trabalhar com maiores carga de sólido.
\end{abstract}

\section{INTRODUÇÃO}

Devido à crescente demanda por energia e principalmente a crescente preocupação com a emissão de gases do efeito estufa e a dependência do petróleo, tem-se aumentado o interesse pelo uso de fontes de energia limpas e renováveis.

No Brasil a cana-de-açúcar é a principal fonte de energia renovável respondendo em 2012 por $15,4 \%$ da oferta total. O etanol de cana substitui quase $40 \%$ das necessidades de gasolina do país, tornando este um combustível alternativo no Brasil e 3\% da necessidade no mundo (Sugarcane, 2014; Ferreira-Leitão et al., 2010). Em 2012/2013 a safra da cana-de-açúcar foi estimada em 588,5 milhões de toneladas (ÚNICA, 2014), o que gerou cerca de 82,4 milhões de toneladas de bagaço (base seca). Aproximadamente a metade desse subproduto foi utilizada na queima direta para geração de calor e energia a fim de suprir a demanda energética do processo. $O$ restante poderia, em tese, ser utilizado para produção de etanol aumentando assim sua produção por tonelada de cana colhida (Biswas et al., 2014). 


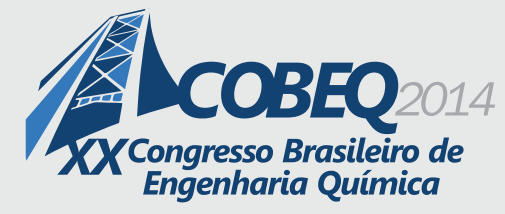

Os materiais lignocelulósicos, como o bagaço de cana-de-açúcar, são complexos orgânicos de carbono cuja estrutura é principalmente representada pela interação físico-química entre a celulose (polímero linear formado por moléculas de glicose ligadas através de ligações $\beta$-1,4-glicosídicas, unidos entre si por ligações inter e intra-moleculares de hidrogênio), hemicelulose (heteropolímero altamente ramificado formado principalmente por pentoses) e a lignina (macromolécula aromática de alto peso molecular) (Ferreira-Leitão et al., 2010; Santos e Gouveia, 2009).

A conversão de materiais lignocelulósicos em açúcares fermentescíveis pode ser feitas por duas rotas tecnológicas: (a) hidrólise ácida, e (b) hidrólise enzimática. A via enzimática apresenta entre outras vantagens: menor consumo de energia, uma vez que opera sob condições brandas de temperatura, $\mathrm{pH}$ e pressão. Além disso, essa rota é altamente específica e não forma produtos de degradação, como furfural e hidroximetilfurfural que causam efeitos indesejáveis durante a etapa de fermentação (Pereira et al., 2011; Ferreira-Leitão, et al., 2010).

Apesar das vantagens apresentadas pela hidrólise enzimática sua aplicação em grande escala ainda esbarra em dificuldades técnicas e econômicas. Entre as estratégias para a viabilidade desta etapa em larga escala estão o aumento da carga de substrato, diminuição da carga enzimática, redução do tempo de hidrólise e a busca por enzimas mais eficientes (Ouyang et al., 2013).

O aumento da carga de substrato é importante a fim de se obter concentrações mais elevadas de açúcares no hidrolisado (Wang et al., 2012; Puri et al., 2013). Esse fator é importante para se obter na etapa de fermentação vinhos com maior teor de etanol, o que reduz o consumo de energia na etapa de destilação (Modenbach e Nokes, 2013).

No entanto, a utilização de altas concentrações de sólidos leva a uma diminuição da conversão do substrato. Esse efeito pode ser explicado por fatores como, dificuldade em se homogeneizar a mistura, inibição da enzima com o aumento da concentração de glicose, problemas de transferência de massa, diminuição da disponibilidade de água, ligação irreversível da enzima ao substrato incluindo a ligação não produtiva com a lignina e desnaturação da enzima (Puri et al., 2013).

Dentro deste contexto o objetivo nesse trabalho foi avaliar o efeito da carga de sólidos na hidrólise enzimática do bagaço de cana-de-açúcar e comparar a eficiência entre dois complexos enzimáticos comerciais.

\section{MATERIAL E MÉTODOS}

\subsection{Material}

Para a avaliação do efeito da carga de sólidos sobre a hidrólise enzimática foi utilizado bagaço de cana-de-açúcar (BCA) in natura cedido pela Usina Iracema (Iracemápolis, SP).

Dois complexos enzimáticos comerciais, denominados complexo-A e complexo-B, foram avaliados nos experimentos. 


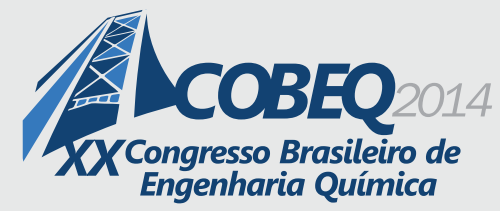

\subsection{Métodos}

Caracterização das amostras: As amostras de bagaço in-natura e pré-tratada foram caracterizadas quimicamente de acordo com procedimento descrito por Gouveia et al., (2009).

Pré-tratamento hidrotérmico: O pré-tratamento hidrotérmico foi realizado em reator de aço inoxidável (modelo 4532, Parr Instruments Company, Moline, Illinois, EUA). A amostra de bagaço de cana-de-açúcar in-natura foi previamente peneirada para separar as partículas grosseiras em peneira de 10 mesh. Em seguida foi submetida à temperatura de $195^{\circ} \mathrm{C}$ por 10 min e $200 \mathrm{rpm}$. Ao final da reação a fração sólida foi separada por filtração e lavada com água para remoção dos componentes solubilizados.

Hidrólise enzimática: A hidrólise enzimática do bagaço pré-tratado foi realizada em meio contendo tampão citrato $(50 \mathrm{mM}, \mathrm{pH} 4,8)$ em um volume total de reação de $50 \mathrm{ml}$. As cargas de sólido avaliadas foram: 10,15 e $20 \%$. O bagaço pré-tratado e o tampão foram adicionados a frascos de Erlenmeyers de $250 \mathrm{~mL}$ e transferidos para o shaker $\left(250 \mathrm{rpm}, 50^{\circ} \mathrm{C}\right)$, por $30 \mathrm{~min}$ até atingir o equilíbrio térmico. Após esse período, as reações de hidrólise foram iniciadas pela adição de enzima (complexos enzimáticos comerciais A e B). A mesma carga enzimática foi empregada em todos os ensaios (20 FPU/gagaço $)$. Todos os ensaios foram realizados em triplicata.

Para quantificação dos açúcares em solução as amostras foram diluídas, filtradas (filtros de $0,45 \mu \mathrm{m})$ e em seguida analisadas por cromatografia líquida de alta eficiência (CLAE). Para a separação foi utilizada a coluna Biorad Aminex HPX-87H na temperatura de $65^{\circ} \mathrm{C}$, fase móvel $\mathrm{H}_{2} \mathrm{SO}_{4}$ $5 \mathrm{mM}$ na vazão $0,6 \mathrm{~mL} / \mathrm{min}$ e detector de índice de refração.

\section{RESULTADOS E DISCUSSÃO}

A Tabela 1 apresenta a caracterização química das amostras de bagaço de cana-de-açúcar (BCA) antes e após a etapa de pré-tratamento hidrotérmico.

Tabela 1 - Caracterização química do BCA antes e após pré-tratamento

\begin{tabular}{cccc}
\hline \hline Componente (\%) & In $_{\text {natura }}$ *** $^{*}$ & Pré-Tratamento-195 $^{\circ} \mathbf{C}$ / 10 min & Perda/Remoção \\
\hline Celulose & 42,4 & 61,2 & 12,8 \\
Hemicelulose & 24,4 & 5,9 & 85,4 \\
Lignina & 24,9 & 31,0 & 24,8 \\
Cinzas & 2,1 & 1,2 & 65,5 \\
Extrativos & 5,5 & - & - \\
Total & 99,3 & 99,3 & - \\
Rendimento mássico & - & 60,4 & - \\
\hline \hline
\end{tabular}

* Rendimento mássico $=\left(\mathrm{m}_{\text {final amostra após pré-tratamento (BS) }} / \mathrm{m}_{\text {inicial amostra }(\mathrm{BS})}\right)$ x 100

*** Fonte: Silva (2011) 


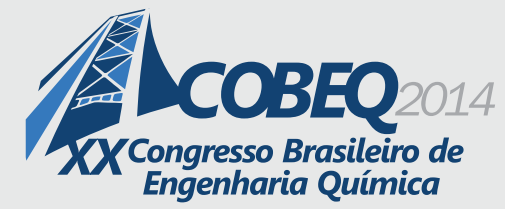

Após o pré-tratamento $85,4 \%$ da hemicelulose e $24,8 \%$ da lignina foram removidos. O prétratamento hidrotérmico é caracterizado por apresentar solubilização de grande parte da hemicelulose (Petersen et al., 2009; Alvira et al.; 2010), com pouca perda/remoção de celulose e lignina. Uma boa remoção da hemicelulose pode contribuir para a ação das enzimas celulolíticas uma vez que essa estrutura forma uma barreira física ao redor da celulose dificultando o acesso das enzimas (Diaz et al., 2010).

Os perfis de concentração de glicose em função do tempo de hidrólise para as cargas de sólidos e os complexos enzimáticos avaliados são apresentados na Figura 1.

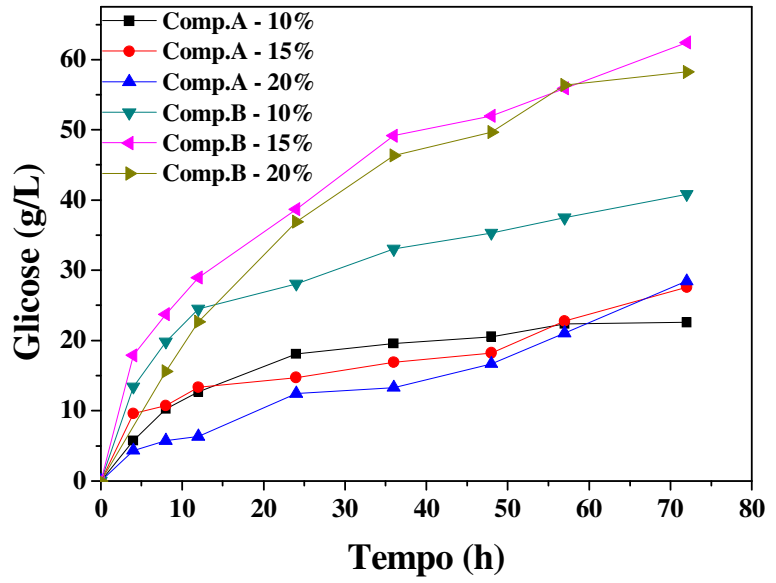

Figura 1- Concentração de glicose em função do tempo nas hidrólises enzimáticas para diferentes cargas de sólidos $(10 \%, 15 \%$ e $20 \%)$ utilizando os complexos enzimáticos A e B.

Observou-se durante a hidrólise enzimática do bagaço pré-tratado um perfil típico da concentração de glicose. Há um rápido aumento da concentração de glicose no início do processo até 10 horas de reação. A influência da carga de sólidos na velocidade inicial de hidrólise é observada para os dois complexos enzimáticos avaliados. Os experimentos realizados com $15 \%$ de carga de sólidos apresentaram maiores velocidades iniciais de reação (observado pelo rápido aumento da concentração de glicose nas primeiras $10 \mathrm{~h}$ de reação) para os dois complexos enzimáticos.

Foram obtidas para as cargas de sólido de $10 \%, 15 \%$ e $25 \%$ as concentrações finais de glicose de 22,6, 27,6, 28,5 g/L (complexo enzimático-A) e 40,9, 62,4 e 58,2 g/L (complexo enzimático B). Comportamento semelhante foi observado por López-Linares et al. (2014) em experimentos com palha de colza e por Ramachandriya et al. (2013) em experimentos utilizando como substrato redcedar oriental (espécie de coníferas).

A Figura 2 ilustra a conversão de celulose em glicose ao longo de 72 horas de hidrólise. Observa-se a redução da conversão com o aumento da carga de sólidos. Esse comportamento foi observado por vários autores (López-Linares et al., 2014; Kristensen, et al., 2009; Ramachandriya, et al., 2013 e Cara et al., 2007). 


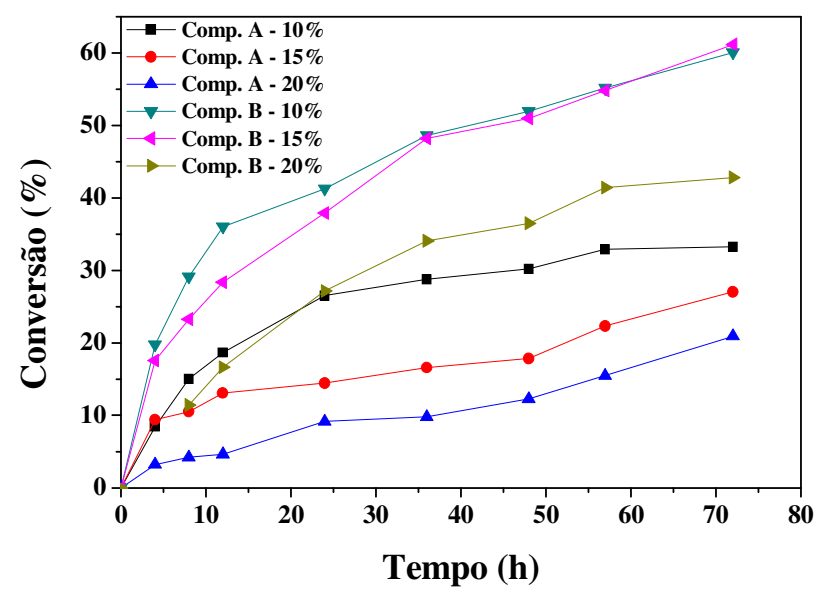

Figura 2- Conversão de celulose em glicose em função do tempo para diferentes cargas de sólidos $(10 \%, 15 \%$ e $20 \%)$ utilizando os complexos enzimáticos A e B.

O aumento da carga de sólidos de 10 para $20 \%(\mathrm{~m} / \mathrm{v})$ levou a uma redução na conversão enzimática de 37 e $29 \%$ para os complexos enzimáticos A e B, respectivamente. Vários fatores podem contribuir para redução da capacidade de conversão pela enzima. Citam-se, entre eles, a inibição da enzima pelos produtos formados (glicose e celobiose), as alterações no substrato e as limitações de transferência de massa (Manazares et al., 2011). Ramachandriya et al. (2013) observaram que a adição de esferas ao meio reacional melhorou a mistura possibilitando o aumento da conversão em experimentos com alta carga de sólidos.

O efeito da carga de sólidos na conversão (em 72 horas) é apresentado na Figura 3.

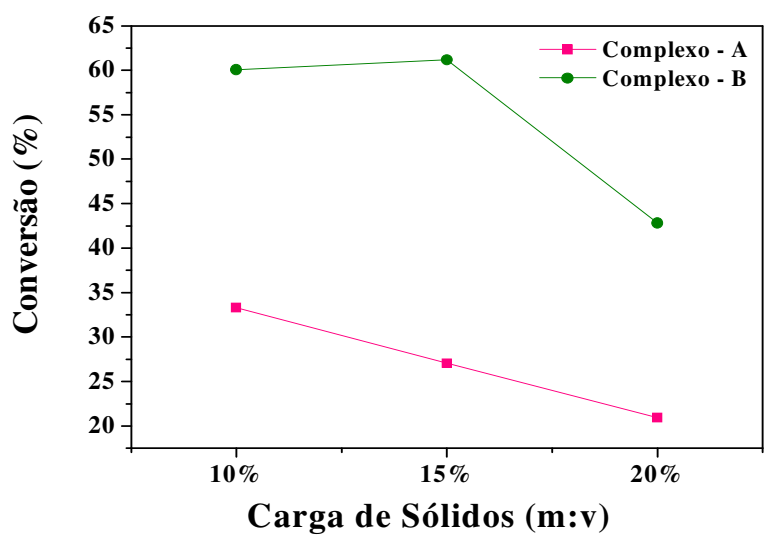

Figura 3- Conversão da celulose em glicose em função da carga de sólido (10\%, 15\% e 20\%) para os complexos enzimáticos A e B.

Observa-se também uma maior eficiência do complexo enzimático-B em relação ao complexoA. Essa maior eficiência pode ser explicada pelo fato de alguns complexos enzimáticos estarem sendo 


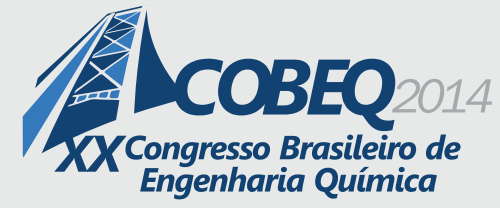

Engenharia Química
19 a 22 de outubro de 2014

Florianópolis/SC

complementados com enzimas oxidantes. Verificou-se que essas enzimas são capazes de clivar ligações $\beta$-glicosídicas fornecendo novos pontos de entrada para as enzimas hidrolíticas (Cannella et al., 2012).

\section{CONCLUSÃO}

Os resultados experimentais mostraram que o aumento da carga de sólidos ocasionou um aumento da concentração final de glicose. No entanto observou-se uma redução da conversão da celulose em glicose durante a hidrólise do bagaço com o aumento da carga de sólidos. Desta forma, como a concentração de glicose ao final da etapa hidrólise é importante (de forma a possibilitar a obtenção de vinhos com maiores teores alcoólicos) deve-se encontrar um equilíbrio entre a carga de sólidos e a conversão durante a etapa de hidrólise. Neste trabalho, nas condições avaliadas, o experimento realizado com $15 \%$ sólidos foi o que resultou na melhor condição. O complexo enzimático-B mostrou desempenho superior ao complexo enzimático-A, permitindo que se trabalhe com carga de sólidos de $15 \%$.

\section{Agradecimentos}

À CAPES, ao CNPq e à FAPESP pelo auxílio financeiro para realização deste trabalho.

\section{REFERÊNCIAS BIBLIOGRÁFICAS}

ALVIRA, P.; TOMÁS-PEJO, E.; BALLESTEROS, M.; NEGRO, M. J. Pretreatment Technologies for an efficient bioethanol production process based on enzymatic hydrolysis: A review. Bioresour. Technol., v. 101, p. 4851-4861, 2010.

BISWAS, R.; UELLENDAHL, H.; AHRING B. K. Wet explosion pretreatment of sugarcane bagasse for enhanced enzymatic hydrolysis. Biomass and Bioenerg., v. 61, p. 104-113, 2014.

CANNELLA, D.; HSIEH, C.; FELBY, C.; JORGENSEN, H. Production and effects of aldonic acids during enzymatic hydrolysis of lignocelluloses at high dry matter content. Biotechnol Biofuels, 5:25, 2012.

CARA, C.; MOYA, M.; BALLESTEROS, I.; NEGRO, M. J.;GONZÁLEZ, A.; RUIZ, E. Influence of solid loading on enzymatic hydrolysis of steam explode dor liquid hot water pretreated olive tree biomass. Process Biochemistry, v. 42, p. 1003-1009, 2007.

DÍAZ, M. J.; CARA, C.; RUIZ, E.; ROMERO, I.; MOYA, M.; CASTRO, E. Hydrothermal prétreatment of rapeseed straw. Bioresour. Technol., v. 101, p. 2428-2435, 2010.

FERREIRA-LEITÃO, V.; GOTTSCHALK, L. M. F.; FERRARA, M. A.; NEPOMUCENO, A. L.; MOLINARI, H. B. C.; BOM, E. P. S. Biomass Residues in Brazil: Availability and Potential Uses. Waste Biomass Valor, v. 1, p. 65-76, 2010.

GOUVEIA, E. R.; NASCIMENTO, R. T.; SOUTO-MAIOR, A. M.; ROCHA, G. J. M. Validação de metodologia para a caracterização química de bagaço de cana-de-açúcar. Quím. Nova, v. 32, p. 1500-1503, 2009. 
KRISTENSEN, J. B.; FELBY, C.; JORGENSEN, H. Yield-determining factors in high-solids enzymatic hydrolysis of lignocellulose. Biotechnol Biofuels. 2:11, 2009.

LÓPEZ-LINARES, J. C.; ROMERO, I.; CARA, C.; RUIZ, E.; MOYA, M.; CASTRO, E. Bioethanol production from rapeseed straw at high solids loading with different process configurations. Fuel, v. 122, p. 112-118, 2014.

MANZANARES, P.; NEGRO, J. M.; OLIVA, J. M.; SAÉZ, F.; BALLESTEROS, I.;CARA, C.; CASTRO, E.; RUIZ, E. Different process configurations for bioethanol production from pretreated olive pruning biomass. J. Chem. Technol. Biotechnol., v. 86, p. 881-887, 2011.

MODENBACH, A. A.; NOKES, S. E. Enzymatic hydrolysis of biomass at high-solids loadings - A review. Biomass and Bioenerg., v. 56, p. 526-544, 2013.

OUYANG, J.; BAOTIAN, L.; ZHANG, M.; ZHENG, Z., YU, HENG. Enzymatic hydrolysis, adsorption, and recycling during hydrolysis of bagasse sulfite pulp. Bioresour. Technol., v. 146, p. 288-293, 2013.

PEREIRA, L. T. C.; PEREIRA, L. T. C.; TEIXEIRA, R. S. S.; BOM, E. P. S.; FREITAS, S. P. Sugarcane bagasse enzymatic hydrolysis: rheological data as criteria for impeller selection. $J$ Ind Microbiol Biotechnol, v. 38, p. 901-907, 2011.

PETERSEN, M. O.; LARSEN, J.; THOMSEN, M. H. Optimization of hydrothermal pretreatment of wheat straw for production of bioethanol at low water consumption without addition of chemicals. Biomass and Bioenerg., v. 33, p. 834-840, 2009.

PURI, J. D.; HEAVEN, S.; BANKS, C. J. Improving the performance of enzyme in hydrolysis of high solid paper pulp derived from MSW. Biotechnol Biofuels, 6:107, 2013.

RAMACHANDRIYA, K. D.; WILKINS, M.; ATIYEH, H. K.; DUNFORD, N. T.; HIZIROGLU, S. Effect of high dry solids loading on enzymatic hydrolysis of acid bisulfate pretreated Eastern redcedar. Bioresource Technol, v. 147, p. 168-176, 2013.

SANTOS, J. R. A.; GOUVEIA, E. R. Produção de bioetanol de bagaço de cana-de-açúcar. Rev Bras de Produtos Agroindustriais, v. 11, n. 1, p. 27-33, 2009.

SILVA, G. M. Pré-tratamento do bagaço de cana-de-açúcar com amônia aquosa para a produção de etanol. 2011. 125 f. (Dissertação), Universidade Federal de São Carlo - UFSCar, São Carlos, 2011.

SUGARCANE.ORG - Brazil' Diverse Energy- Matrix. Disponível em: <http://sugarcane.org/thebrazilian-experience/brazils-diverse-energy-matrix > Acesso em: 20/04/2014.

ÚNICA - Histórico de Produção e Moagem-Por Safra. Disponivel em: $<\mathrm{http} / / /$ www.unicadata.com.br/historico-de-producao-e-moagem.php?idMn=32\&tipoHistorico=4> Acesso em: 20/04/2014.

WANG, L.; TEMPLER, R.; MURPHY, R. J. High-solidsloading enzymatic hydrolysis of waste papers for biofuel production. Appl Energy, v. 99, p. 23-31, 2012. 